\title{
Vertical Cooperative Advertising: A Manufacturer's Perspective
}

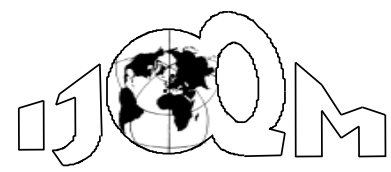

DOI: $10.46970 / 2020.26 .3 .1$

Volume 26, Number 3

September 2020, pp. 163-182

\author{
Lihong He \\ Lanzhou University \\ (helh@1zu.edu.cn)
}

Ilhyung Kim

Zhe Gerorge Zhang

Western Washington University

(ilhyung.kim@wwu.edu)

(george.zhang@wwu.edu)

Vertical cooperative advertising, where a manufacturer participates in his retailer's local advertising, has been examined extensively in the operations research literature. Yet there are some controversies in the literature regarding when such cooperative advertising is beneficial. We attempt to reconcile these controversies by considering all relevant variables in a single framework. We find that the manufacturer's participation in local advertising is a reasonable strategy for him only when the following conditions are met: The retail price is driven by the market, the manufacturer's contribution margin is high compared to the retailer's, and the demand is inelastic or moderately elastic.

Keywords: Supply Chain Coordination, Vertical Cooperative Advertising, Pricing Decision, Stackelberg Game

\section{Introduction}

In a traditional supply chain setting, all members in the supply chain work independently as a buyer, a seller or both. Such an independent practice, however, often results in inferior performance for the entire supply chain since the members are primarily concerned with optimizing their own objectives. To achieve better performance, various types of collaboration strategies among the supply chain members have emerged in practice such as buyback contracts, revenue sharing, options contracts, vendor-managed inventory control, third party logistics, and cooperative advertising. Among them, cooperative advertising has attracted particular attention from industry as well as academia for the past decades. (For concepts, strategies, and cases on cooperative advertising, see Young and Greyser 1983; for industrial practices and empirical findings on cooperative advertising, see Dutta et al. 1995; and for literature reviews on cooperative advertising, see Aust and Buscher 2014.) Cooperative advertising is an arrangement of sharing the advertising costs of a supply chain among its members. Technically, any members can share the costs with any other members, whether they are in the same echelon or a different echelon in the supply chain. One of the most common practices of cooperative advertising is vertical cooperative advertising, where a manufacturer offers to pay a certain fraction of his retailer's expenditure on locally placed advertising. Dutta et al. (1995) investigated 2,156 cooperative advertising arrangements and found that such arrangements represented a significant portion of the marketing activities of firms. 
They also found that the participation rate, the proportion of local advertising that a manufacturer shares with his retailer, varied from 25 to 100 percent with 74.6 percent being the average. According to Borrell Associates (2015), co-op advertising programs in North America had grown 20 percent annually from 2012 to 2015. These programs were expected to reach $\$ 36$ billion in 2015.

The cooperative advertising has been extensively studied in academia as well. Aust and Buscher (2014) provided a comprehensive review of the articles on cooperative advertising. They found 110 publications on this topic dated as early as 1970, of which 58 scientific articles examined vertical cooperative advertising. Most of them have been published since 2000, predominantly in the operational research literature. The number of previous studies and the recent trend clearly show an increased interest in vertical cooperative advertising. This paper also studies this topic, especially from the perspective of a manufacturer. In the rest of this section, we first summarize some key findings of the previous studies, and then highlight the contributions of this paper beyond the already discovered results.

In vertical cooperative advertising, the most important variable is obviously the manufacturer's participation in retailer's local advertising. More specifically, it is critical to find out whether such participation would be beneficial to the manufacturer and, if yes, under what conditions and how intensely the manufacturer should participate in the local advertising. Berger (1972), in his pioneer work on vertical cooperative advertising, introduced a mathematical model for a two-level supply chain consisting of a retailer and a manufacturer. He determined the optimal advertising allowance given to the retailer by the manufacturer, and demonstrated, through numerical examples, that his quantitative analysis could yield significantly better solutions than a simple 50-50 split between the manufacturer and the retailer, which was back then commonly used in practice. While Berger (1972) considered a single type of advertising, perhaps local advertising placed by the retailer, Huang and $\mathrm{Li}$ (2001) considered not only the retailer's local advertising but also the manufacturer's global or brand advertising. The manufacturer's global advertising is intended to develop brand recognition and consumer royalty in the long run. Thus, it may not necessarily lead to an immediate increase in sales. On the other hand, the retailer's local advertising seeks to stimulate consumers' buying behavior through more targeted approaches. As a result, it may have a direct promotional effect on sales in the short run. Huang and $\mathrm{Li}$ (2001) found that when the manufacturer was the leader in a Stackelberg game (more details on the Stackelberg game will be discussed in the next section), he should offer an advertising allowance to the retailer if his profit margin was large. When the manufacturer was not the leader or there was no leader in the channel, the manufacturer should not offer any advertising allowance. They also found that the manufacturer's advertising allowance, if provided, was positively correlated to the retailer's local advertising expenditure, i.e., the manufacturer could induce the retailer to invest more on local advertising by providing an allowance to her. Yue et al. (2006) added a new variable to the model developed by Huang and Li (2001). They considered a price discount provided by the manufacturer directly to the consumers. Yue et al. (2013) further added another variable, a retailer's price discount to the consumers. Both studies found similar results comparable to Huang and $\mathrm{Li}$ (2001). Instead of using the price discounts, Szmerekovsky and Zhang (2009) examined the advertising and the pricing decisions 
at the same time. They considered the retail and wholesale prices as the decision variables in addition to the local and global advertising expenditures and the manufacturer's participation rate in the local advertising. Contrary to the above findings and the common practices observed in various industries, Szmerekovsky and Zhang (2009) showed that the manufacturer should not support the local advertising in any circumstances; instead, he should consider lowering the wholesale price or focusing on the global advertising. Note that their model assumes that the demand is elastic. Using a different demand function, Xie and Neyret (2009), Xie and Wei (2009), and Seyed Esfahani, Biazaran, and Gharakhani (2011) still found that it would be beneficial for the manufacturer to participate in local advertising and, by doing so, induce the retailer to invest more in local advertising. The readers are referred to Aust and Buscher (2014) for other findings. It is apparent, from the above disparate results, that the optimal solutions heavily depend on the underlying models; specifically, the decision variables available for the manufacturer and the retailer and the types of functions assumed for demand.

In this paper, we attempt to reconcile the above mentioned controversies appearing in the previous studies by developing a model, which simultaneously considers all of the previously studied decision variables in a single framework and captures all three types of price elasticity: elastic, unit-elastic, and inelastic demand. We also examine the trade-offs among the decision variables and their behaviors with respect to the system parameters. Specifically, we investigate the following research questions:

1. Should the manufacturer participate in his retailer's local advertising? Some previous studies recommended yes while some others recommended no. We attempt to clarify these controversies by systematically examining the conditions under which such participation is beneficial to the manufacturer.

2. In addition to participating in local advertising, the manufacturer can increase demand by performing a broad scale advertising campaign, lowering the wholesale price, or providing a price discount directly to the consumers. Under what conditions should the manufacturer focus on each of these strategies or a combination of them? How would the manufacturer's decisions be affected by the parameters in the model such as price and advertising elasticities?

3. Are the strategies for the manufacturer reinforcing or balancing each other? We examine inter-relationships for all possible pairs of the manufacturer's different strategies. As an example, should a larger price discount be combined with a larger or smaller advertising? Should the manufacturer invest more in global advertising when he subsidizes the retailer's local advertising?

4. How would the manufacturer's decisions affect the retailer's behavior? We consider interactions between the manufacturer's and the retailer's decisions. As an example, would an advertising allowance by the manufacturer induce the retailer to invest more in local advertising? Would a price discount offered by the manufacturer directly to the consumers make the retailer increase or decrease the retail price?

The rest of the paper is organized as follows. Section 2 provides the details of our model. We also highlight where our model stands compared to the existing models in the literature. In Section 3, we discuss the retailer's responses to the manufacturer's decisions in the manufacturer Stackelberg game. We then examine in Section 4, based on the retailer's responses, the relationships among the decision variables, 
which lead us to develop two subsequent models. In the Section 5, we provide the analytical results of the two models. Finally, we conclude the paper with some suggestions for future research in Section 6.

\section{A Generalized Model}

To make our model comparable to the previously studied models, we consider a twotier supply chain consisting of a manufacturer and a retailer, which is the predominant composition in the literature. There are other compositions studied in the literature, which considered the competition among multiple manufacturers and/or multiple retailers. These are important extensions and may result in different implications than the above bilateral monopoly. The competitive settings, however, restrict the decision space or make it difficult to obtain analytical solutions. As an example, Xiao et al. (2019) examined a two-tier chain with a single manufacturer and $n$ retailers; however, they assumed that the retail and wholesale prices were given parameters rather than decision variables; otherwise, they would not be able to solve their model analytically. For the foregoing reasons, we focus on the bilateral monopoly, which allows us to consider virtually all decision variables examined in the previous studies.

The manufacturer produces a product with the manufacturing cost $\kappa$ and sells the product to the retailer with the wholesale price $w$. The retailer sells the product to the consumers with the retail price $p$. Thus, we have $0<\kappa<w<p$. In order to increase the demand, the manufacturer offers a price discount $d$ directly to the consumers. Since it is unrealistic to provide a discount more than the manufacturer's contribution margin, we have $0 \leq d<w-\kappa$. The retailer spends $a$ in local advertising and the manufacturer spends $q$ in global advertising. In addition to the above five decision variables ( $w, p, d, a$, and $q$ ), we allow the manufacturer to subsidize the retailer's local advertising. Let $t(0 \leq t<1)$ represent the participation rate, the proportion of retailer's local advertising subsidized by the manufacturer. Thus, the manufacturer spends $t a$ in local advertising and $q$ in global advertising, while the retailer spends $(1-t) a$ in local advertising and none in global advertising. Though it is not common, some manufacturers subsidize the entire cost of local advertising (i.e., $t=1$ ). In this case, the local advertising expenditure controlled by the retailer will be infinity since it does not cost the retailer at all. To avoid this unrealistic situation, the manufacturers set the accrual rate, the maximum amount of expenditure that the manufacturers will pay. As examined in Dutta et al. (1995), when participation rates were less than 100 percent, the participation rates were most likely binding, in other words, the accrual rates would not affect anyone's decisions. When participation rates were set at 100 percent, the accrual rates were binding. Thus, in most cases, we do not need to consider both rates, and just one of them is sufficient. We consider the participation rate, which is one of the core variables in the cooperative advertising literature.

Zhang, Xie, and Chen (2013) considered not only the manufacturer's participation in local advertising but also the retailer's participation in global advertising. This is an interesting extension. In this mutual collaboration setting, however, the manufacturer was restricted to control either his global advertising expenditure or his participation in local advertising, but not both. Otherwise, as the authors pointed out, the optimization problem would result in a trivial or unreasonable solution. 
Furthermore, the mutual collaboration has not been found in any industrial practices. Due to the restricted decision space and the lack of relevance, we do not consider the retailer's participation in global advertising.

In addition to the above advertising and pricing decisions, Karray and Surti (2016) examined two other decision variables: the retailer's order quantity and the quantity discount offered by the manufacturer. They assumed that the retail price was endogenous, i.e., determined by the order quantity and advertising expenditures, and further assumed that the demand was the same as the order quantity. Zhou, Li, and Zhong (2018) also examined the order quantity as a decision variable under uncertain demand and analyzed underage and overage costs in a newsvendor-type model. They considered the retail and wholesale prices as parameters rather than decision variables. The order quantity and the quantity discount are important decision variables in inventory management. However, due to the mathematical complexity, adding inventory-related variables does not allow us to keep other important variables such as the retail and wholesale prices. Setting aside the mathematical complexity, it may distort our analyses from important managerial implications if we combine the strategic level decisions (advertising and pricing decisions) with the tactical level decisions (operations and inventory decisions) in a single aggregate model. For the foregoing reasons, we do not consider operational decisions such as the order quantity, quantity discount, and other inventory-related decisions in our model.

In sum, we consider the following six decision variables: the retail and wholesale prices, the retailer's local and manufacturer's global advertising, the manufacturer's participation rate in local advertising, and the price discount provided by the manufacturer directly to the consumers. Note that the retailer's price discount to the consumers is not necessary once the retail price is considered as a decision variable. Thus, our model includes all relevant decision variables examined in the previous models. Furthermore, our model is also comprehensive compared to other models in terms of demand functions, which will be discussed next.

The demand functions used in the cooperative advertising literature can be categorized into two approaches. In the first or static approach, a steady-state demand is assumed, which depends on parameters and variables in a given function but is independent of time. In the second or dynamic approach, the demand rate depends on time in addition to other parameters and variables. As discussed earlier, the global advertising focuses on a long-run effect, while the local advertising emphasizes an immediate or short-run effect. The dynamic approach, in general, is more appropriate to differentiate these two types of advertising by capturing timedependent demand using differential equations. The static approach, however, has advantages over the dynamic approach. It makes the corresponding analyses more flexible, and as a result, allows us to examine the behaviors of decision variables more thoroughly. We can still differentiate the two types of advertising using different parameters within the static approach. For the foregoing reasons, we adopt the static approach. We focus on finding important managerial implications based on the trade-offs among the various decision levers that the manufacturer can play with. It certainly will be an interesting extension of this paper to consider the dynamic nature of the two different types of advertising using the dynamic approach. 
We now discuss the static demand functions used in the existing literature and highlight where our model stands compared to them. Virtually all demand functions used in the previous studies depend on the local and global advertising expenditures (or efforts) and the retail price. We refer to the demand as a function of advertising as the advertising demand function and the demand as a function of price as the price demand function. As recognized in Aust and Buscher (2014), a square root function and a power function are the two most commonly used functions in the literature for the advertising demand function. The square root function assumes that the effects of local and global advertising are additive, while the power function assumes that they are multiplicative. Though a static demand function is not as systematic as a dynamic demand function to differentiate the effects of local and global advertising, in order to attain such differentiation within the scope of the static approach, the power function is more appropriate than the square root function due to its flexibility of choosing different exponents and the interaction or synergy effect given in the multiplicative form. Both the power and the square root functions are concave, and this property is required for the existence of the optimal advertising levels. The square root function assumes that the demand increases indefinitely as the advertising increases, while the power function used in the literature assumes a saturation point or an upper bound, beyond which the demand cannot increase any further. Due to this saturation point, the power function could become negative for a certain range of parameters. This problem was, in fact, recognized by Yue at al. (2006). Ahmadi-Javid and Hoseinpour (2012) imposed a constraint to avoid the negative demand associated with the power function. In this paper, we consider a different form of power function for the advertising demand function. Our advertising demand function does not assume a saturation point and, as a result, it does not generate negative demand. More specifics on our advertising demand function will be discussed later in this section.

Regarding the price demand function, a linear function, which is widely used in the marketing literature, is also very widely used in modeling cooperative advertising since it simplifies the subsequent mathematical analyses. Another commonly used function is a power function, where the elasticity of demand is depicted by the exponent. Obviously, it is more general to assume the power function since the linear function is basically a special case of the power function. We also use a power function in this paper. The power functions examined in the literature had some limitations: Szmerekovsky and Zhang (2009) considered a power function, but they assumed that the elasticity was greater than one or the demand was elastic. Yue et al. $(2006,2013)$ considered a power function with all three levels of price elasticity, but they assumed that the demand was a function of the price discount rather than the price itself. In this case, even if the price increases or decreases, the demand will remain unchanged as long as the percentage discount, the ratio of the price discount to the original price, is maintained. Unlike the price demand function in the literature, we consider a more comprehensive power function, which depends on the price (not the percentage discount) and exhibits all three levels of price elasticity.

Below is the demand function considered in this paper

$Q(a, q, p, d)=\beta a^{\mu} q^{\nu}(p-d)^{-\gamma}$, 
where $\mu, v$, and $\gamma$ are positive constants representing the quasi-elasticities of local and global advertising and the price elasticity, respectively. As shown in (1), the demand is jointly affected by the local and global advertising and the price observed by the consumers. The price elasticity $\gamma$ determines whether the demand is inelastic $(0<\gamma<1)$, unit-elastic $(\gamma=1)$, or elastic $(\gamma>1)$. To ensure the convex feature of the demand function, we can further restrict the quasi-elasticities to be $0<\mu<1$ and $0<v<1$. These restrictions are consistent with the previous models in the literature. The positive constant $\beta$ can be referred to as the base demand when $a=q=p=1$ and $d=0$. Note that the advertising expenditures $a$ and $q$ are not restricted and can take any positive values.

Finally, using the demand function given in (1) and the decision variables available for the players in the supply chain, the profit functions of the manufacturer, the retailer, and the entire supply chain, are determined, respectively, as follows

$$
\begin{aligned}
& M(w, q, t, d)=(w-d-\kappa) \beta a^{\mu} q^{\nu}(p-d)^{-\gamma}-t a-q, \\
& R(p, a)=(p-w) \beta a^{\mu} q^{v}(p-d)^{-\gamma}-(1-t) a, \\
& T(p, w, a, q, t, d)=(p-d-\kappa) \beta a^{\mu} q^{v}(p-d)^{-\gamma}-a-q .
\end{aligned}
$$

Regarding the nature of cooperation among the channel members, Aust and Buscher (2014) categorized the previous studies into three game-theoretic scenarios: a non-cooperative simultaneous game (Nash equilibrium), a non-cooperative sequential game (Stackelberg equilibrium), and a centralized coordination (cooperative equilibrium). The Nash equilibrium is used to determine noncooperative and simultaneous decisions of two or more channel members. On the other hand, the Stackelberg equilibrium determines non-cooperative and sequential decisions, where one player, as the leader, first makes his or her decisions, and then all others act like a follower and subsequently make decisions according to the leader's choices. The cooperative equilibrium determines the jointly optimized decisions among all players as if they are completely coordinated by a single decision maker. Among these different game-theoretic settings, Aust and Buscher (2014) observed that the most prevalent setting in vertical cooperative advertising is the manufacturer Stackelberg game in a bilateral monopoly, where the manufacturer has the channel leadership and the retailer acts as the follower. In this paper, we also focus on the manufacturer Stackelberg game to make our results comparable to previous findings. Furthermore, this game is the most adequate setting to analyze the manufacturer's perspective since it provides the broadest decision space for the manufacturer. We neither consider the cooperative equilibrium nor the Nash equilibrium in this paper. It is clear that the cooperative equilibrium provides the highest profit for the entire supply chain. However, in this scenario, we do not need to consider the manufacturer's participation in local advertising since who pays for the advertising does not matter. The Nash equilibrium scenario is not relevant to our study either since the manufacturer, in this scenario, will not offer any advertising allowance to the retailer. 


\section{Retailer's Responses to Manufacturer's Decisions}

In the manufacturer Stackelberg game, the manufacturer first determines his optimal decisions, and then the retailer makes her optimal decisions according to the manufacturer's decisions. We now examine how the retailer reacts to the manufacturer's decisions. The retailer maximizes her profit given in (3). Thus, we can get the retailer's optimal solutions by differentiating (3) with respect to her two choices, the retail price $p$ and the local advertising expenditure $a$.

Theorem 1: In the manufacturer Stackelberg game, for given manufacturer's decisions $w, q$, $t$, and $d$, the optimal retail price and the optimal local advertising expenditure are

$$
\begin{aligned}
& p^{*}=\left\{\begin{array}{cc}
\frac{\gamma}{\gamma-1} w-\frac{1}{\gamma-1} d, & \text { if } \gamma>1 \\
p^{\max }, \quad \text { if } 0<\gamma \leq 1
\end{array}\right. \text { and } \\
& a^{*}=\left\{\begin{array}{cc}
\left(\frac{\beta \mu(\gamma-1)^{\gamma-1} q^{\nu}}{\gamma^{\gamma}(1-t)(w-d)^{\gamma-1}}\right)^{\frac{1}{1-\mu}}, & \text { if } \gamma>1 \\
\left(\frac{\beta \mu q^{\nu}\left(p^{\max }-w\right)}{(1-t)\left(p^{\max }-d\right)^{\gamma}}\right)^{\frac{1}{1-\mu}}, & \text { if } 0<\gamma \leq 1
\end{array}\right.
\end{aligned}
$$

respectively, where $p^{\max }$ is the maximum retail price allowed by the market.

Proof: See Appendix A-1. (All appendices will be available upon request from the authors.)

When the demand is elastic $(\gamma>1)$, there exists an optimal retail price as shown in (5). However, when the demand is inelastic or unit-elastic $(0<\gamma \leq 1), \partial R / \partial p>0$, i.e., the higher the retailer price, the higher the retailer's profit. In this case, without any further restrictions, the retailer could increase her profit indefinitely, which is obviously unrealistic. Thus, we assume that the retail price in this case is driven by the market, i.e., the retailer will charge the maximum price allowed by the market.

From Theorem 1, we can make the following observations on how the retailer responds to the manufacturer's decisions:

1. When the demand is elastic $(\gamma>1)$, the optimal retail price depends on the wholesale price. The retailer increases (or decreases) her retail price more than the amount by which the manufacture raises (or lowers) his wholesale price. It is interesting that the retailer's reaction to a change in the wholesale price is greater than the change itself, whether the wholesale price is raised or lowered.

2. When $\gamma>1$, if the manufacturer offers a discount to the consumers, the retailer further decreases her retail price. The amount of decrease in the retail price is greater than, equal to, or less than the discount when $1<\gamma<2, \gamma=2$, or $\gamma>2$, respectively. Thus, the manufacturer's offering of a discount to the consumers tends to be more effective when the demand is moderately elastic $(1<\gamma<2)$ than when it is highly elastic $(\gamma \geq 2)$. 
3. When the demand is inelastic or unit-elastic $(0<\gamma \leq 1)$, the optimal retail price is not affected by the manufacturer's decisions. The retailer charges the maximum price allowed by the market.

4. Unlike the optimal retail price, the optimal local advertising expenditure depends on manufacturer's all four decision variables regardless of the price elasticity. The retailer spends more in local advertising as the manufacturer increases his subsidy toward it, increases his spending in global advertising, lowers the wholesale price, or offers more discounts to the consumers. In other words, as the manufacturer moves in favor of the retailer's profit, the retailer reacts more actively to increase the demand through her local advertising. Contrary to our finding, all the previous studies mentioned in the first section showed either negative or no relationship between the retailer's local advertising and the manufacturer's global advertising. These somewhat counter-intuitive results are related to their demand functions. The advertising demand functions of the previous studies were either a power function with an upper bound or an additive square-root function. In the case of the power function with an upper bound, as the global advertising $q$ increases, the demand gets closer to the bound and, as a result, the effectiveness of the local advertising $a$ diminishes. Thus, the relationship between $a$ and $q$ is likely to be negative. In the case of the additive square-root function, due to the additive (rather than multiplicative) structure, $a$ and $q$ are independent from each other. Thus, there is no relationship between the two advertising expenditures. On the other hand, our demand function is multiplicative and does not exhibit an upper bound. Accordingly, the relationship between $a$ and $q$ turns out to be positive. We, of course, do not claim that our model is right and others are wrong. We understand that different relationships are possible depending on how the demand is determined by the local and global advertising. The inconsistency observed above emphasizes how important it would be to set up a mathematical model in a stylized modelling approach.

It is straightforward to obtain the above observations from the first derivatives of the retailer's two optimal decisions with respect to the manufacturer's four variables. For complete analyses of the above observations, see Appendix A-2.

\section{Two Reduced Models}

In this section, we first discuss the relationships among the decision variables, and then propose two reduced models based on the relationships. From (5), we can easily obtain the price realized by the consumers as follows

$$
p^{*}-d=\left\{\begin{array}{ll}
\frac{\gamma(w-d)}{\gamma-1}, & \text { if } \gamma>1 \\
p^{\max }-d, & \text { if } 0<\gamma \leq 1
\end{array} .\right.
$$

In addition, the profit margin for the retailer is determined as 


$$
p^{*}-w=\left\{\begin{array}{ll}
\frac{w-d}{\gamma-1}, & \text { if } \gamma>1 \\
p^{\max }-w, & \text { if } 0<\gamma \leq 1
\end{array} .\right.
$$

From (7) and (8), we notice that, when the demand is elastic, manufacturer's two decision variables, the wholesale price $w$ and the price discount $d$, are identical in terms of their impacts on the price realized by the consumers and the retailer's profit margin. Furthermore, from (6), we also realize that, when the demand is elastic, the two decision variables, $w$ and $d$, are identical in terms of their impact on the advertising expenditure spent for local advertising. The above findings lead us to the following conclusion:

Theorem 2: In the manufacturer Stackelberg game, when the demand is elastic, the manufacturer's and the retailer's profits, the demand, and the price realized by the consumers will be the same whether the manufacturer reduces the wholesale price by a certain amount or he provides a price discount directly to the consumers by the same amount.

Proof: We omit the details.

When the demand is elastic, since the impacts of $w$ and $d$ are identical, only one of them should be included in the model as a decision variable. On the other hand, when the demand is inelastic or unit-elastic, the retail price is assumed to be determined by the market, i.e., it is bounded by $p^{\max }$. In this case, if we allow the manufacturer to determine his own wholesale price, he would make it as high as possible and the retailer's profit would be zero. To avoid this impractical situation, we further assume that the wholesale price is also driven by the market. Thus, when the demand is not elastic, both $p$ and $w$ are no longer the decision variables. Finally, the original model with six decision variables can be reduced, depending on the price elasticity, to the following two models:

Model 1: When the demand is elastic $(\gamma>1)$, the original model can be reduced to a five-variable model with $w$ (or $d$ ), $q, t$ for the manufacturer and $p$ and $a$ for the retailer.

Model 2: When the demand is inelastic or unit-elastic $(0<\gamma \leq 1)$, the original model can be reduced to a four-variable model with $d, q, t$ for the manufacturer and $a$ for the retailer. Note that, in this case, the impacts of $d$ on the demand and profits are no longer identical to those of $w$. Thus, $d$ should be considered as a decision variable.

In addition to the above two cases, it would be interesting if we extend Model 2 to the case when the demand is elastic and the prices $p$ and $w$ are determined by the market. The two reduced models including the extension are summarized in Table 1, which consists of $2 \times 2$ grid depending on whether or not the demand is elastic (columns) and how the retail and wholesale prices are determined (rows). The top right corner of the grid, where the demand is not elastic and the prices can be chosen freely by the players, would not be realistic in its current form unless some further 
restrictions are imposed on the demand or the prices. We will analyze the two models in the next section.

Table 1 Two Reduced Models based on Price Elasticity and Price Determination

\begin{tabular}{|l|c|c|}
\hline & Demand: Elastic, $\gamma>1$ & $\begin{array}{c}\text { Demand: Inelastic or } \\
\text { unit-elastic, } 0<\gamma \leq 1\end{array}$ \\
\hline $\begin{array}{l}\text { Prices } p \text { and } w \text { : Freely chosen by the players } \\
\text { in a supply chain }\end{array}$ & Model 1 & Unrealistic \\
\hline Prices $p$ and $w$ : Driven by the market & Model 2 & Model 2 \\
\hline
\end{tabular}

\subsection{Model 1}

\section{Optimal Decisions of the Two Reduced Models}

We now discuss Model 1 when the retail and wholesale prices are freely chosen by the retailer and the manufacturer, respectively. As shown in Table 1, this model is valid only when the demand is elastic. In the manufacturer Stackelberg game, by the backward approach, we first solve the retailer's problem for a given set of manufacturer's decisions, and then solve the manufacturer's problem. The retailer's responses to the manufacturer's decisions for the generalized model have been discussed in Section 3. By substituting the retailer's optimal responses in (5) and (6) into the manufacturer's profit function in (2), we can jointly determine the manufacturer's optimal decisions. Note that, in Model 1, the price discount is not included in the manufacturer's decision variables. Thus, we have $d=0$ in (2), (5), and (6) when analyzing this model. The manufacturer's optimal decisions are given in the following theorem:

Theorem 3: In the manufacturer Stackelberg game, when the demand is elastic $(\gamma>1)$, the optimal participation rate, the optimal wholesale price, and the optimal global advertising expenditure are

$t^{*}=0$

$w^{*}=\frac{\gamma-\mu}{\gamma-1} \kappa$, and

$$
q^{*}=\left\{\begin{array}{cc}
\left(\frac{\beta \mu^{\mu} v^{1-\mu}(\gamma-1)^{2 \gamma-\mu-1}}{\kappa^{\gamma-1} \gamma^{\gamma}(\gamma-\mu)^{\gamma-\mu}}\right)^{\frac{1}{1-\mu-\nu}}, & \text { if } \nu<1-\mu \\
q^{\max }, & \text { if } \nu>1-\mu \text { or } \\
& v=1-\mu \text { and } \frac{\beta \mu^{\mu}(\gamma-1)^{2 \gamma-\mu-1}}{\kappa^{\gamma-1} \gamma^{\gamma}(\gamma-\mu)^{\gamma-\mu}}>\left(\frac{1}{1-\mu}\right)^{1-\mu} \\
0, & \text { if } v=1-\mu \text { and } \frac{\beta \mu^{\mu}(\gamma-1)^{2 \gamma-\mu-1}}{\kappa^{\gamma-1} \gamma^{\gamma}(\gamma-\mu)^{\gamma-\mu}}<\left(\frac{1}{1-\mu}\right)^{1-\mu}
\end{array}\right.
$$


respectively, where $q^{\max }$ is the maximum global advertising expenditure allowed by a budget or other constraint. Furthermore, the optimal retail price and the optimal local advertising expenditure given in (5) and (6) in Theorem 1 can be rewritten using the model parameters only (without the decision variables) as follows

$$
\begin{aligned}
& p^{*}=\frac{\gamma(\gamma-\mu)}{(\gamma-1)^{2}} \kappa \text { and } \\
& a^{*}=\left\{\begin{array}{cc}
\left(\frac{\beta \mu^{1-\nu} v^{\nu}(\gamma-1)^{2 \gamma+\nu-2}}{\kappa^{\gamma-1} \gamma^{\gamma}(\gamma-\mu)^{\gamma+\nu-1}}\right)^{\frac{1}{1-\mu-\nu}}, & \text { if } v<1-\mu \\
\left(\frac{\beta \mu(\gamma-1)^{2 \gamma-2}\left(q^{\max }\right)^{\nu}}{\kappa^{\gamma-1} \gamma^{\gamma}(\gamma-\mu)^{\gamma-1}}\right)^{\frac{1}{1-\mu}}, & \text { if } v>1-\mu \text { or } \\
v, & \text { if } v=1-\mu \text { and } \frac{\beta \mu^{\mu}(\gamma-1)^{2 \gamma-\mu-1}}{\kappa^{\gamma-1} \gamma^{\gamma}(\gamma-\mu)^{\gamma-\mu}}>\left(\frac{1}{1-\mu}\right)^{1-\mu} \frac{\beta \mu^{\mu}(\gamma-1)^{2 \gamma-\mu-1}}{\kappa^{\gamma-1} \gamma^{\gamma}(\gamma-\mu)^{\gamma-\mu}}<\left(\frac{1}{1-\mu}\right)^{1-\mu}
\end{array} .\right.
\end{aligned}
$$

Proof: See Appendix B-1.

From Theorem 3, we make the following observations on the manufacturer's optimal decisions:

1. When the demand is elastic, contrary to the common practices in vertical cooperative advertising, the manufacturer should not participate in local advertising $\left(t^{*}=0\right)$. Instead, he should lower his wholesale price, spend more on global advertising, or provide a price discount directly to the consumers. This result is consistent with Szmerekovsky and Zhang (2009), but not consistent with all other studies in vertical cooperative advertising introduced in the first section. We categorize them into two groups depending on potential reasons for the inconsistencies. The first group treated the retail and wholesale prices as given parameters rather than decision variables (Huang and Li 2001; Yue et al. 2006, 2013). The second group treated the prices as decision variables, but they assumed that the demand was either a linear function of the retail price or an additive square-root function of the advertising expenditures (Xie and Wei 2009; Xie and Neyret 2009; SeyedEsfahani et al. 2011). The inconsistencies among the previous studies with different models/assumptions underscore how critical it would be, in a modeling approach, to set up the functional form of a model and restrict its decision space.

2. At $t=t^{*}$ and $w=w^{*}$, the manufacturer's profit function is concave, linear, or convex in $q$ depending on the quasi-elasticity of global advertising. As shown in (11), when the profit function is concave $(v<1-\mu)$, there exits an optimal global advertising expenditure (the first solution). When it is convex $(v>1-\mu)$ or linear $(v=1-\mu)$ and increasing, the manufacturer's profit could increase indefinitely as $q$ increases. Though we have not explicitly considered in this paper, there should be a budget or other type of constraint, 
which would limit the expenditure. Thus, $q^{*}=q^{\max }$, where $q^{\max }$ is the maximum allowed by the constraint (the middle solution). When it is linear and decreasing, the global advertising is not profitable. Thus, $q^{*}=0$ (the last solution).

3. Using the solutions in (11) and (13) when $v<1-\mu$, we can get the ratio of the optimal local advertising expenditure to the optimal global advertising expenditure as follows

$$
\frac{a^{*}}{q^{*}}=\frac{\mu}{v}\left(\frac{\gamma-\mu}{\gamma-1}\right)
$$

The ratio decreases (i.e., the global advertising becomes more effective than the local advertising) as the price elasticity or the quasi-elasticity of global advertising increases. With respect to the quasi-elasticity of local advertising $\mu$, as it increases, the ratio first increases until $\mu$ reaches $\gamma / 2$, and then decreases beyond that point.

4. By analysing the behaviour of optimal solutions with respect to system parameters, we can determine whether the optimal solutions are reinforcing or balancing each other. In Model 1, the manufacturer has three levers: $t, w$, and $q$. Among them, the optimal participation rate $t^{*}=0$, so the only relationship that can be examined is the one between the optimal wholesale price $w^{*}$ and the optimal global advertising expenditure $q^{*}$. With respect to $\kappa$, the two optimal solutions always move in the opposite direction. On the other hand, with respect to $\gamma$ and $\mu$, they may move in the same or opposite direction depending on the system parameters. Let us explain these results in managerial implications. To provide a more plausible implications, we will use $d^{*}$ instead of $w^{*}$. Note that, as specified in Theorem 2, increasing $d$ by one unit is equivalent to decreasing $w$ by one unit. As $\kappa$ increases, it becomes more difficult for the manufacturer to provide a discount. Thus, he should reduce the discount and, at the same time, he also needs to reduce the global advertising to maximize his profit. A small $d^{*}$ should be paired with a small $q^{*}$ and vice versa. With respect to the price elasticity $\gamma$ and quasi-elasticity of local advertising $\mu$, as they increase, the demand becomes more sensitive to the price or the local advertising level. Thus, the manufacturer should provide more discount resulting in a higher $d^{*}$. Should he also increase the global advertising? The answer to this question is not straight forward, it could be yes or no depending on system parameters. More precise analyses on the conditions for reinforcing and balancing relationships are given in Appendix B-2. These results emphasize how complicated it would be to make the strategic decisions in vertical cooperative advertising.

The above observations can be obtained from the first derivatives of the optimal decisions with respect to other variables and parameters. For complete analyses, see Appendix B-2. 


\subsection{Model 2}

We now consider Model 2 when the retail and wholesale prices are driven by the market. As shown in Table 1, this model is valid when the demand is not only elastic but not elastic as well. Model 2 has four decision variables: three variables $(q, t$, and $d$ ) for the manufacturer and one variable $(a)$ for the retailer. The profit functions given in (2), (3) and (4) are still valid, but $p$ and $w$ are no longer the decision variables. Since the profit function for the retailer and its first derivative with respect to the local advertising $a$ are the same as the original model, the optimal local advertising expenditure is, in fact, the same as the lower solution of (6). The optimal solution is reproduced below. Note that Model 2 treats $p$ and $w$ as parameters rather than decision variables.

$$
a^{*}=\left(\frac{\beta \mu q^{v}(p-w)}{(1-t)(p-d)^{\gamma}}\right)^{\frac{1}{1-\mu}}
$$

By substituting (15) into the manufacturer's profit function in (2), we can determine the manufacturer's optimal decisions.

Theorem 4: In the manufacturer Stackelberg game, when the retail and wholesale prices are driven by the market, the manufacturer's optimal discount offered to the consumers and the optimal participation rate in local advertising are as follows

$$
\begin{aligned}
& t^{*}=\left\{\begin{array}{cc}
\frac{(2-\gamma-\mu)(p-w)+\kappa}{(1-\mu)(p-w)+\kappa}, & \text { if } p<p_{0} \text { and } \gamma_{d}<\gamma<\gamma_{t} \\
\frac{w-\kappa-(1-\mu)(p-w)}{w-\kappa+\mu(p-w)}, & \text { if } p<p_{0} \text { and } \gamma \leq \gamma_{d} \\
0, & \text { otherwise }
\end{array}\right. \text { and } \\
& d^{*}=\left\{\begin{array}{cc}
\frac{\gamma(w-\kappa)+\gamma \mu(p-w)-p}{\gamma-1}, & \text { if } p<p_{0} \text { and } \gamma_{d}<\gamma<\gamma_{t} \\
\frac{\gamma(w-\kappa)-(1-\mu) p}{\gamma-1+\mu}, & \text { if } p<p_{0} \text { and } \gamma \geq \gamma_{t} \text { or } p \geq p_{0} \text { and } \gamma>\gamma_{0} \\
0, & \text { otherwise }
\end{array}\right.
\end{aligned}
$$

where $p_{0}=w+(w-\kappa) /(1-\mu), \quad \gamma_{d}=p /(w-\kappa+\mu(p-w)), \quad \gamma_{t}=2-\mu+\kappa /(p-w)$, and $\gamma_{0}=p(1-\mu) /(w-\kappa)$. Based on whether or not $d^{*}$ and $t^{*}$ are positive, four different situations can be constructed. For each of the four situations, the manufacturer's optimal global advertising expenditure is determined as follows: 


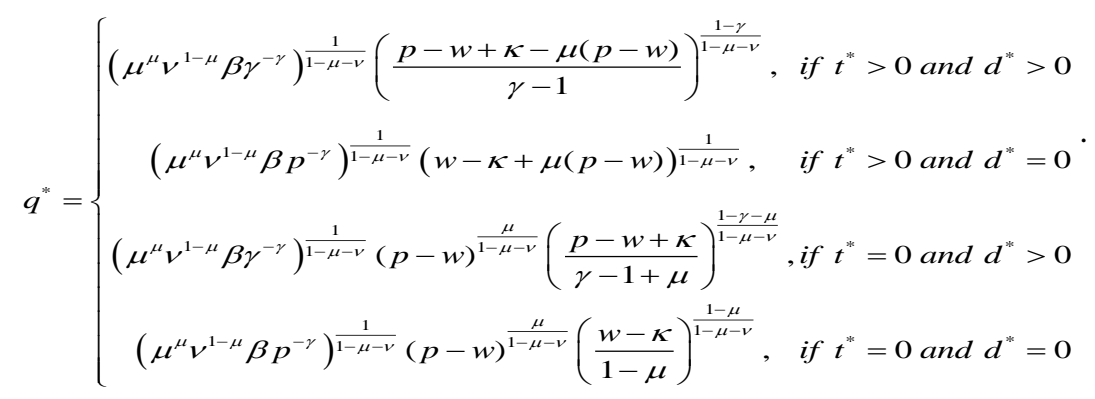

Furthermore, the retailer's optimal local advertising expenditures given in (15) can be rewritten for the four situations as follows

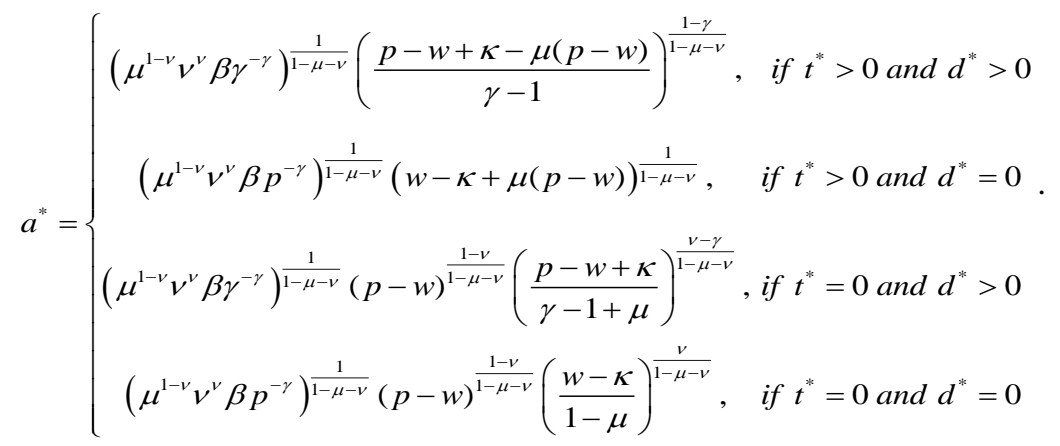

\section{Proof: See Appendix C-1.}

From Theorem 4, we make the following observations on the manufacturer's optimal decisions:

1. As discussed in Theorem 1, the retailer spends more in local advertising as the manufacturer increases his subsidy toward it. Thus, the manufacturer is willing to provide a subsidy to the retailer if the additional demand generated by the increased local advertising is large enough to compensate the subsidy. To satisfy this condition, the advertising elasticity $\mu$ should be high or equivalently the price elasticity should be lower than $\gamma_{t}$.

2. Likewise, the manufacturer is willing to provide a discount to the consumers if the additional demand generated by the discount is large enough to compensate the reduction in revenue due to the discount. To satisfy this condition, the price elasticity should be higher than $\gamma_{d}$.

3. Both $\gamma_{t}$ and $\gamma_{d}$ are greater than one, and their relative magnitude depends on the wholesale and retail prices and the manufacturing cost. If $p<p_{0}$ or equivalently $(w-\kappa)>(1-\mu)(p-w)$, in other words, the manufacturer's contribution margin is greater than $(1-\mu)$ times the retailer's contribution margin, then $\gamma_{d}<\gamma_{t}$; otherwise, $\gamma_{d} \geq \gamma_{t}$. The optimal solutions for these two cases are separately explained in next two observation points.

4. When $p<p_{0}$, depending on the price elasticity, the decision space can be divided into three regions: $0<\gamma \leq \gamma_{d}, \gamma_{d}<\gamma<\gamma_{t}$, and $\gamma \geq \gamma_{t}$. Figure 1 depicts 
the manufacturer's optimal decisions on $t$ and $d$ for the three regions. If the price elasticity is low $\left(\gamma \leq \gamma_{d}\right)$, it is more effective for the manufacturer to participate in local advertising rather than providing a discount to the consumers, i.e., $t^{*}>0$ but $d^{*}=0$. On the other hand, if the price elasticity is $\operatorname{high}\left(\gamma \geq \gamma_{t}\right)$, the manufacturer's optimal decisions will be the exact opposite, i.e., $t^{*}=0$ but $d^{*}>0$. If the price elasticity is in the middle $\left(\gamma_{d}<\gamma<\gamma_{t}\right)$, the manufacturer should use both levers, the participation in local advertising and the price discount, i.e., $t^{*}>0$ and $d^{*}>0$.

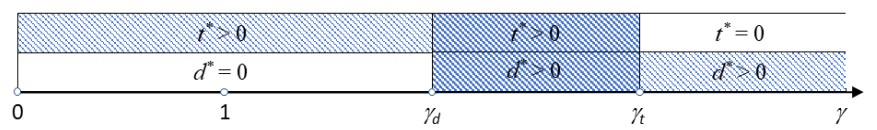

Figure 1 Manufacturer's Decisions on tand $d$ when $p<p_{0}$ in Model 2

5. When $p \geq p_{0}$, we have $\gamma_{t} \leq \gamma_{d} \leq \gamma_{0}$. Unlike the previous case when $p<p_{0}$, the two strategies ( $d$ and $t$ ) never overlap, i.e., the manufacturer should not use both strategies at the same time. Furthermore, the retail price in this case is too high (or equivalently the wholesale price is too low relative to the retail price) to have a positive $t^{*}$. Thus, as shown in Figure 2, the decision space is divided into two regions depending on the price elasticity. The manufacturer should turn off both $t$ and $d$ until the price elasticity reaches $\gamma_{0}$ and, beyond this point, he should consider providing a discount to the consumers.

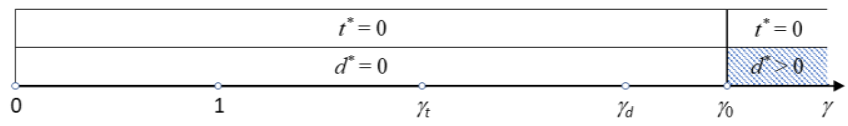

Figure 2 Manufacturer's Decision on $t$ and $d$ when $p \geq p_{0}$ in Model 2

6. When both $t^{*}$ and $d^{*}$ are positive, the two optimal decisions move in exact opposite direction with respect to system parameters $\gamma, \mu$ and $\kappa$. Specifically, as the price elasticity increases, the quasi-elasticity of local advertising increases, or the manufacturing cost decreases, the optimal participation rate $t^{*}$ decreases while the optimal price discount $d^{*}$ increases. In other words, a small $t^{*}$ should be paired with a large $d^{*}$ and vice versa. Figure 3 illustrates this trade-off when the price elasticity $\gamma$ varies from 0 to 3 . In this numerical example, we set: $p=5$, $w=4, \kappa=1$, and $\mu=0.67$.

7. The nature of the relationship between $t^{*}$ and $q^{*}$ is not monotonic; it can be either reinforcing or balancing depending on the system parameters. As an example, as the price elasticity increases, the optimal participation rate $t^{*}$ decreases, while the optimal global advertising initially decreases until rreaches a certain point and then increases. Thus, $t^{*}$ and $q^{*}$ reinforce each other when $\gamma$ is small, while they balance each other when $\gamma$ is large. In other words, a large subsidy offered to the retailer should be paired with a large global advertising when the price elasticity is small, but with a small global advertising when it is large. With respect to other parameters, $\kappa$ and $\mu$, both reinforcing and balancing relationships are observed between $t^{*}$ and $q^{*}$. More details are given in Appendix C-2. 


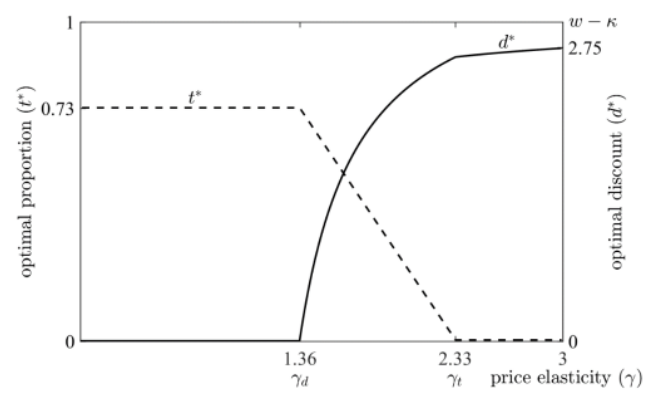

Figure 3 The Optimal Participation Rate and the Optimal Price Discount when $p<p_{0}$ in Model 2

8. We already observed that $d^{*}$ and $t^{*}$ move in exact opposite direction with respect to parameters $\gamma, \mu$ and $\kappa$. Thus, the relationship between $d^{*}$ and $q^{*}$ is exact opposite to the relationship between $t^{*}$ and $q^{*}$ just discussed in the previous observation. We omit the details. The above observations emphasize that the manufacturer's optimal decisions should not be determined in a simple way. A careful examination is required to find the manufacturer's optimal decisions because even a small change in one of the parameters could dramatically turn a reinforcing relationship into a balancing one.

9. By combining (18) and (19) when both $t^{*}$ and $d^{*}$ are positive, the ratio of the optimal local advertising expenditure to the optimal global advertising expenditure is obtained as follows

$$
\frac{a^{*}}{q^{*}}=\frac{\mu}{v}
$$

Compared to the same ratio for Model 1 given in (14), the above ratio is quite simple and straightforward. It only depends on the two quasi-elasticities and does not depend on any other parameters including the price elasticity. The ratio for Model 2 is simple because the retail and wholesale prices are treated as fixed parameters in this model.

The above observations can be obtained mostly from the first derivatives of the optimal decisions with respect to other variables and parameters. For complete analyses, see Appendix C-2.

\section{Conclusions and Future Research Directions}

The vertical cooperative advertising has been studied substantially in the past decade. However, there are still disagreements in the previous studies regarding whether such advertising is beneficial to the manufacturer and, if yes, when the manufacturer should provide a subsidy to support his retailer's local advertising. We attempted to resolve these existing discrepancies by developing a comprehensive model under the manufacturer Stackelberg game. We found that the manufacturer's two decision variables, the wholesale price charged to the retailer and the discount 
offered to the consumers, were identical in terms of their impacts on the demand and the profits of the manufacturer and the retailer. Based on this identity and the retailer's response to the manufacturer's decisions, we were able to reduce the original model to two subsequent models which contained a smaller set of decision variables. The analyses of the two reduced models provided many interesting implications. Specifically, we found the conditions under which the manufacturer's participation in his retailer's local advertising was beneficial to him. We also found how the retailer would change her retail price and her spending in local advertising when the manufacturer changed his wholesale price, provided a discount to the consumers directly, offered a subsidy to her local advertising, and invested in global advertising. In addition, we analyzed when the manufacturer's different strategies would reinforce or balance each other.

Our findings are based on a stylized model assuming a simple two-level supply chain. It will be a very valuable addition to this study if we measure or verify the findings empirically using a real dataset. For example, it will be interesting to measure whether the manufacturer's profit improves or deteriorates after participating in vertical cooperative advertising and how such changes are affected by the market and firm characteristics. It would be also interesting to measure how the retailer's pricing and advertising decisions are related to the manufacturer's different strategies examined in this study.

It is known that advertising affects not only the amount of demand but also the elasticity of demand. In our model, both types of advertising, whether global and local, were assumed to affect the amount of demand, but not the elasticity. This assumption might be the reason why the manufacturer's participation in local advertising was not supported when the demand was elastic. If the advertising makes the customers more royal to the products and less sensitive to the changes in price, the vertical cooperative advertising might be a more attractive option to the manufacturer. This extension could be examined by making the price elasticity as a function of advertising.

\section{References}

1. Ahmadi-Javid, A., and Hoseinpour, P. (2012), on a cooperative advertising model for a supply chain with one manufacturer and one retailer, European Journal of Operational Research, Vol. 219 (No. 2), pp. 458-466.

2. Aust, G., and Buscher, U. (2014), Cooperative advertising models in supply chain management: A review, European Journal of Operational Research, Vol. 234 (No. 1), pp. 1-14.

3. Berger, P. D. (1972), Vertical cooperative advertising ventures, Journal of Marketing Research, Vol. 9 (No. 3), pp. 309-312.

4. Borrell Associates (2015), The changing face of co-op programs: Digital media forges new opportunities for brand managers, White paper sponsored by Netsertive, August 2015.

5. Dutta, S., Bergen, M., John, G., and Rao, A. (1995), Variations in contractual terms of cooperative advertising contracts: An empirical investigation, Marketing Letters, Vol. 6 (No. 1), pp. 15-22. 
6. Huang, Z., and Li, S. X. (2001), Co-op advertising models in manufactureretailer supply chains: A game theory approach, European Journal of Operational Research, Vol. 135 (No. 3), pp. 527-544.

7. Karray, S., and Surti, C. (2016), Channel coordination with quantity discounts and/or cooperative advertising, International Journal of Production Research, Vol. 54 (No. 17), pp. 5317-5335.

8. SeyedEsfahani, M. M., Biazaran, M., and Gharakhani, M. (2011), A game theoretic approach to coordinate pricing and vertical co-op advertising in manufacturer-retailer supply chains, European Journal of Operational Research, Vol. 211 (No. 2), pp. 263-273.

9. Szmerekovsky, J. G., and Zhang, J. (2009), Pricing and two-tier advertising with one manufacturer and one retailer, European Journal of Operational Research, Vol. 192 (No. 3), pp. 904-917.

10. Xiao, D., Zhou, Y., Zhong, Y., and Xie, W. (2019), Optimal cooperative advertising and ordering policies for a two-echelon supply chain, Computers \& Industrial Engineering, Vol. 127, pp. 511-519.

11. Xie, J., and Neyret, A. (2009), Co-op advertising and pricing models in manufacturer-retailer supply chains, Computers \& Industrial Engineering, Vol. 56 (No. 4), pp. 1375-1385.

12. Xie, J., and Wei, J. C. (2009), Coordinating advertising and pricing in a manufacturer-retailer chain, European Journal of Operational Research, Vol. 197 (No. 2), pp. 785-791.

13. Young, R. F., and Greyser, S. A. (1983), Managing cooperative advertising: A strategic approach. Lexington, Mass.: Lexington Books.

14. Yue, J., Austin, J., Huang, Z., and Chen, B. (2013), Pricing and advertisement in a manufacturer-retailer supply chain, European Journal of Operational Research, Vol. 231 (No. 2), pp. 492-502.

15. Yue, J., Austin, J., Wang, M. C., and Huang, Z. (2006), Coordination of cooperative advertising in a two-level supply chain when manufacturer offers discount, European Journal of Operational Research, Vol. 168 (No. 1), pp. 6585.

16. Zhang, J., Xie, J., and Chen, B. (2013), Cooperative advertising with bilateral participation, Decision Sciences, Vol. 44 (No. 1), pp. 193-203.

17. Zhou, Y., Li, J., and Zhong, Y. (2018), Cooperative advertising and ordering policies in a two-echelon supply chain with risk-averse agents, Omega, Vol. 75, pp. 97-117.

\section{Acknowledgement}

The authors are grateful to Professor Gerard Campbell at Western Washington University for his valuable suggestions on this paper. This work was supported by the National Natural Science Foundation of China under grant number 71972094; and the Fundamental Research Funds for the Central Universities under grant number 17LZUJBWTD005. 


\section{About Our Authors}

Lihong He is a Professor of Supply Chain Management at the School of Management, Lanzhou University, China. She received her BSc degree in applied mathematics in 1989, her master's degree in computational mathematics in 1999, and $\mathrm{Ph} . D$. in solid mechanics in 2005, all from Lanzhou University, China. She has taught courses on Data, Model and Decisions to MBAs, and Operations Research and Statistics to undergraduates at the School of Management, Lanzhou University. Her research interests are currently focused on pricing and advertising strategies, supply chain management, operations research, and applied statistics.

Ilhyung Kim is a Professor of Decision Sciences at Western Washington University. He holds a Ph.D. in Operations and Technology Management from the University of California, Los Angeles (UCLA). He teaches applied statistics, predictive analytics, and operations management. His current research interests are supply chain coordination, manufacturing and service systems design, and data mining.

Zhe George Zhang is a Professor in Management Science at Western Washington University in the USA and an Adjunct Professor in Operations Research at Simon Fraser University in Canada. He obtained his Ph.D. in Operations Research from the University of Waterloo, Canada. The focus of his current research is on theory and application of queueing and other stochastic models. 